\title{
The Evolution of Microtubule End-Binding Protein 1 (EB1) and Roles in Regulating Microtubule Behavior
}

\author{
Jiayu Liu ${ }^{1,2}$, Rong Han ${ }^{1,2 *}$ \\ ${ }^{1}$ School of Life Science, Shanxi Normal University, Linfen, China \\ ${ }^{2}$ Higher Education Key Laboratory of Plant Molecular and Environmental Stress Response (Shanxi Normal \\ University) in Shanxi Province, Linfen, China \\ Email: 619248095@qq.com,
}

Received 28 July 2015; accepted 22 August 2015; published 27 August 2015

Copyright (C) 2015 by authors and Scientific Research Publishing Inc.

This work is licensed under the Creative Commons Attribution International License (CC BY). http://creativecommons.org/licenses/by/4.0/

(c) (i) Open Access

\begin{abstract}
All organisms must transmit genetic information to offspring through cell division, and mitotic spindle participates in the process. Spindle dynamics through depolymerization or polymerization of microtubules generates the driving force required for chromosome movements in mitosis. To date, studies have shown that microtubule arrays control the directions of cell division and diverse microtubule-associated proteins regulate cell division. But a clear picture of how microtubules and microtubule-associated proteins modulate cell division remains unknown. Depletion of end-binding protein 1 by RNA-mediated inhibition shows that one of the microtubule-associated proteins, end-binding protein 1, plays a crucial role in mitotic spindle formation and promotes microtubule dynamics and is needed for the proper segregation of mitotic chromosomes during anaphase in Drosophila cells. Here, we review the properties of end-binding protein 1 and the roles of end-binding protein 1 in regulating microtubule behavior and in cell cycle.
\end{abstract}

\section{Keywords}

Microtubules, End-Binding Protein 1, Chromosome Segregation

\section{Introduction}

The microtubule cytoskeleton is essential for a variety of essential processes such as intracellular organization, intracellular transport, cell motility, and mitosis of eukaryotic cells. This is possible because of the intrinsic dy-

\footnotetext{
${ }^{*}$ Corresponding author.
}

How to cite this paper: Liu, J.Y. and Han, R. (2015) The Evolution of Microtubule End-Binding Protein 1 (EB1) and Roles in Regulating Microtubule Behavior. American Journal of Plant Sciences, 6, 2114-2121. 
namic properties of microtubules. Microtubules (MTs) are dynamic hollow tubes comprising $\alpha, \beta$-tubulin dimers that disassemble and reassemble at two ends: the slow-growing (minus) and fast-growing (plus) ends. It is now widely known that MT behavior is modulated by a number of MT-associated proteins (MAPs), which can influence dynamic instability parameters and consequently impact on mitotic progression and fidelity. Many of these MAPs share the ability to recognize only the distal part of a polymerizing MT, known as the MT plus end. For this reason, these MAPs are currently known as MT plus-end-tracking proteins (+TIPs) [1] [2]. Recently, +TIPs have emerged as regulators of MT dynamics. The plus end explores the cell periphery and shows dynamic instability, switching rapidly between the two phases of growth and shrinkage. Thus this dynamic scaffold performs a variety of very different functions. Genetic and biochemical studies have shown that +TIPs interact with each other and form protein complexes [3].

End-binding protein 1 (EB1) promotes MT polymerization and interacts directly with many other +TIPs and cytoskeletal proteins such as cytoplasmic linker protein 170 (CLIP-170) and the dynactin large subunit p150 ${ }^{\text {Glued }}$, and mitotic centromere-associated kinesin, microtubule-actin crosslinking factor and adenomatous polyposis coli (APC) [4] [5]. Thus, EB1 has been proposed to form the core of the microtubule plus-end complex and act as a hub in interactions with +TIPs [6] [7].

Division of one cell into two genetically identical daughter cells occurs through two coordinated processes which are known as mitosis (division of the nucleus) and cytokinesis (division of the cytoplasm). The transition from interphase to mitosis involves a dramatic reorganization of the MT cytoskeleton. In fact, there is an increase in MT dynamics which occurs concomitantly with NEB that could be important for spindle morphogenesis [8] [9]. Studies have shown that accurate segregation of the replicated genome during cell division depends on dynamic attachments between kinetochores, proteinaceous structures assembled on the centromeric regions of chromosomes, and spindle microtubules. Kinetochores harness the forces generated by microtubule dynamics to drive chromosome segregation and ensure chromosome biorientation [10]. EB1 localizes to the plus ends of polymerizing MTs, suggesting that it may regulate MT dynamics during mitosis [11]. Depletion of Dm EB1 affects microtubule dynamics but causes minimal perturbation of microtubule organization in interphase cells. This result indicates that Dm EB1 promotes microtubule dynamics in Drosophila cells. Moreover, Dm EB1 is necessary for proper chromosomal segregation and spindle elongation during anaphase [12]. And studies show that together with APC, EB1 regulates chromosomal stability during mitosis [13]. So, EB1 is important for driving chromosome segregation by promoting microtubule dynamics. But a clear picture of how EB1 regulate microtubule behavior to drive chromosome segregation has not still emerged.

\section{Microtubule Structure and Dynamics}

Microtubules are intrinsically dynamic structures. In cells they are usually found in states of either growth or shrinkage and they exhibit rapid transitions between the two phases. This feature, termed dynamic instability, underlies many aspects of microtubule function including the ability to rearrange into different arrays [14] [15]. Microtubules are polymeric cylinders composed of $\alpha$ and $\beta$ tubulin dimers. Rows of tubulin subunits, protofilaments, associate with each other laterally to form the microtubule lattice. Because they are arranged head to tail into 13 protofilaments that are aligned longitudinally in the tubule wall, microtubules are intrinsically polar: the plus-end where $\beta$-tubulin monomer is exposed, is the fast-growing end in vitro and the only end that grows in cells. The opposite (minus) end can slowly grow in vitro, while in cells it is usually stabilized or serves as the site of disassembly [16]. Microtubules are structurally polar and dynamic filaments that grow by addition of guanosine triphosphate (GTP)-loaded tubulin subunits to their end. After complex, largely unknown structural rearrangements at the nanoscale, GTP hydrolysis and phosphate release lead to the formation of a guanosine diphosphate (GDP)-loaded microtubule lattice. The matured microtubule lattice is protected from depolymerization by a stabilizing structure at the growing microtubule end. Stochastic loss of this end structure leads to depolymerization (catastrophe) [17].

In cells, MT dynamics are not only regulated by the intrinsic dynamic instability of the polymers, but also by stabilizing/destabilizing structural MT-associated proteins (MAPs) that play a role in MT organization [18]. Among these, a large number of MAPs specifically recognize the terminal portion of MT. These are collectively known as MT plus-end-tracking proteins or +TIPs [1]. These +TIPs appear as comets in the MT tip, moving throughout the cell as MT grows and disappearing when MT shrinks [19]. One of these +TIPs, EB1 recruits several other proteins to growing microtubule ends and EB1 as a microtubule maturation factor and provide a 
mechanistic explanation for its effects on microtubule growth and catastrophe frequency, which cause microtubules to be more dynamic. EB1 binding accelerates conformational maturation in the microtubule, most likely by promoting lateral protofilament interactions and by accelerating reactions of the guanosine triphosphate (GTP) hydrolysis cycle. The microtubule maturation time is directly linked to the duration of a growth pause just before microtubule depolymerization, indicating an important role of the maturation time for the control of dynamic instability [20].

\section{The Microtubule Plus-End Binding Protein 1 (EB1) and Localization of EB1 in Cells}

The EB1 protein is a member of the exciting and enigmatic family of microtubule (MT) tip-tracking proteins. EB1 acts as an exquisite marker of dynamic MT plus ends in some cases, whereas in others EB1 is thought to directly dictate the behavior of the plus ends [6]. EB1 was the first member identified in a yeast two-hybrid screen as an interactor of the C-terminus of the adenomatous polyposis coli (APC) tumor suppressor protein [21]. EBs are relatively small, elongated proteins (around $32 \mathrm{kDa}$ ) with conserved structural features. All members have at the N-terminal region an MT-binding portion containing a calponin homology $(\mathrm{CH})$ domain with a highly conserved fold [2]. It was shown that this $\mathrm{CH}$ domain was both required and sufficient for binding to MT plus ends [22] [23]. The C-terminal portion of EB1, on the other hand, contains a coiled-coil region which is necessary for EB dimerization. The EEY/F motif at the flexible tail region provides a binding site for CAP-Gly domains found in select cytoskeleton-associated proteins, including a +TIP p150 Glued [4] [24]. Members of the EB1 family (EBs) are mostly known for recruiting a variety of other plus-end-tracking proteins through interactions with their C-terminal EB homology domain [4] [25] [26]. The N-terminal microtubule binding domains bind to the outer microtubule surface in the grooves between adjacent protofilaments, close to the exchangeable GTP binding site [27]. End binding (EB) proteins are part of a highly conserved family which, in mammalians, comprises three members encoded from three different genes: EB1, EB2 (RP1), and EB3 (EB3F). Both EB1 and EB3 seem to be ubiquitously expressed, whereas EB2 expression is restricted to only certain cell types/tissues [28]. Normally, EB1 is expressed in higher levels when compared to other EBs. However, EB3 is also highly expressed in specific cell types. EB3 was originally reported in neurons, where it was shown to interact with a brain-specific form of APC (APC2), but it is also highly abundant in muscle cells [29]. EB1 homologs are also found in plant lineages [30] [31], but interestingly, the EEY/F motif is not present in plant EB1 proteins, which are divided in two subgroups. Arabidopsis has three EB1s: EB1a, EB1b, and EB1c [32]. EB1a and EB1b are similar in sequence and localize to MT plus ends in all MT structures throughout cell cycle. EB1c, which is conserved only in vascular plants, localizes to nucleoplasm during interphase and colocalizes with MT only in the mitotic spindle and phragmoplast [11] [33] [34]. Both immunofluorescence analyses and live imaging using GFP tagging showed that EB1 is able to localize to the growing ends of MTs throughout mitosis [8] [35]. More in-depth observations demonstrated that EB1 can target to kinetochores with attached growing MTs [36]. Besides its plus end localization, EB proteins were also shown to bind other subcellular structures either directly (centrosome) or indirectly (F-actin and membranes). The functions for the $\mathrm{CH}$ and CC domains are conserved in the Arabidopsis EB1 proteins. The C-terminal tail of the EB1a and EB1b subgroup is autoinhibitory in the MT assembly capacity of EB1, despite the absence of the EEY/F motif; instead, the EB1c tail has a nuclear localization signal (NLS). EB1a and EB1b form heterodimers with each other, but not with EB1c. Furthermore, the EB1genes are expressed in various cell types of Arabidopsis, but the expression of EB1c is particularly strong in the meristematic cells where it is targeted to the nucleus by a nuclear localization signal in the C-terminal tail [11].

\section{Roles of EB1 in Regulating Microtubule Behavior}

The dynamic properties of microtubules are regulated by multiple proteins. Two of them, EB1 and XMAP215 (chTOG in humans), are special in that they accumulate autonomously at microtubule ends [37]. EB1 is selective for growing ends (not distinguishing between plus and minus ends). And recruits several other proteins to growing microtubule ends and has seemingly antagonistic effects on microtubule dynamics [38] [39]. The first report regarding the possible role of EB proteins in MT dynamics came from the observation that, when overexpressed, these proteins induced the formation of acetylated MT bundles that were resistant to nocodazole treatment [40]. In addition, their ability to tip-track MTs led to the possibility that they might be involved in MT dy- 
namics regulation, particularly in promoting MT growth [41]. This was confirmed in many independent studies using not only different model organisms such as budding and fission yeast, Drosophila, and human cells, but also in vitro systems [23] [42] [43]. Growing microtubule ends serve as transient binding platforms for essential proteins that regulate microtubule dynamics and their interactions with cellular substructures. End-binding proteins (EBs) autonomously recognize an extended region at growing microtubule ends with unknown structural characteristics and then recruit other factors to the dynamic end structure. The calponin homology $(\mathrm{CH})$ domain of the fission yeast EB Mal3 bridges protofilaments except at the microtubule seam. By binding close to the exchangeable GTP-binding site, the $\mathrm{CH}$ domain is ideally positioned to sense the microtubule's nucleotide state. The same microtubule-end region is also a stabilizing structural cap protecting the microtubule from depolymerization. This supports that there is a common structural link between microtubule dynamic instability and end tracking [27]. EB1 senses conformational changes within the microtubule lattice induced by reactions taking place as part of the GTP hydrolysis cycle [27] [44]. This leads to the well-known comet-like accumulation of EBs at the end region of growing microtubules where high-affinity binding sites are gradually lost with time [45]. The impact of EB proteins on interphase MT dynamics may also involve their interaction with other +TIPs. In fact, differences in the expression and regulation of several +TIPs in different cell types may be responsible for the observed differences in specific MT populations [46]. In addition, data derived from in vitro assays demonstrated that EB1 can act cooperatively with other + TIPs such as CLIP170 in the regulation of MT dynamics. Accordingly, it was demonstrated that both EB1 and CLIP170 can synergize to modulate MT dynamics, possibly by modifying the MT-stabilizing cap [47]. Moreover, EB association with CLASPs was also reported to affect MT dynamics at the cell cortex by increasing MT rescue events [48]. Interestingly, EBs can also associate with and load MT depolymerizers such as MCAK to the MT plus ends [49]. This interaction is important for the localization of MCAK to the plus ends but also to enhance its catastrophe-inducing activity. Thus, by allowing the accumulation of polymerizers and depolymerizers at the MT plus end, EB proteins facilitate the rapid switching between MT growth and shortening. The overall picture that has emerged confirms the role of EB proteins in the regulation of MT dynamics, but their precise effect is still not fully understood. Recent work showed that modulation of MT dynamics by EB proteins can also be regulated by phosphorylation. In budding yeast, the single EB-like protein was described to be phosphorylated by Ipl1p/Aurora-B and this is important to regulate the association of EB to spindle MTs [50]. Moreover, a mutation in the fission yeast EB-like protein was sufficient to increase MT binding, leading to their stabilization [51]. In humans, less is known about the phosphorylation of EB proteins. Recent work demonstrated that EB3 is phosphorylated by Aurora kinases on S176 during mitosis [52]. This Aurora-mediated EB3 phosphorylation leads to a significant increase in MT growth, allowing stabilization of the midbody [53]. Dephosphorylation of EB3 restricts cortical MT growth, allowing proper daughter cell adhesion to the substrate. Inversely, phosphorylation of EB3 on S162 by the Src-PLCg2 signaling pathway was shown to block MT growth, leading to adherens junction stabilization in interphase cells [54]. Taken together, these data demonstrate that EB protein association to the MT plus ends can be regulated by phosphorylation, although it is still unclear how different phosphorylation events integrate to control EB function, thus regulating MT dynamics in different tissues.

\section{Roles of Microtubule and EB1 in Cell Cycle}

Proper regulation of MT (microtubule) dynamics is essential for various vital processes, including the segregation of chromosomes, directional cell migration and differentiation. Spindle dynamics through depolymerization or polymerization of microtubules (MT) generates the driving force required for chromosome movements in mitosis [55]. MT assembly and disassembly is modulated by a complex network of intracellular factors that cooperate or antagonize each other, are highly regulated in space and time and are thus attuned to the cell cycle and differentiation processes [56]. Cell-cycle progression is accompanied by changes in MT dynamics at very specific stages. This is accompanied by an increase in MT dynamics and an abrupt decrease in MT polymer level which tightly correlates with NEB [57]. So studies of roles of microtubule and EB1 are important to understand the life of plants.

Interactions between microtubules plus-end and the cell cortex are important for accurate positioning of the spindle [58]. During normal mitosis, the mitotic spindle positions itself at the geometric center of the cell. In S2 cells lacking Dm EB1, however, the spindle was frequently mis-positioned. This shows that mitotic spindle positioning requires EB1 activity. At the same time, the Loss of Dm EB1 function causes defects in mitotic spindle 
structure in metaphase cells that could be classified into four general categories. The most common defect was a complete loss of astral microtubules. The second class of defects lacked astral microtubules and exhibited an overall compaction of the spindle into a basket-like meshwork of microtubules surrounding the chromosomes. The third type of defect was a detachment of a spindle pole from the bundles of microtubules that were connected to the kinetochores. The fourth category of defect was "barrel-shaped" spindles that maintained their symmetry, but failed to focus the microtubules at the poles and also lacked astral microtubules [12]. So, will these prevent normal chromosome segregation during mitosis? Whether chromosome segregation will be abnormal in these cases remains unclear. Dm EB1 plays a crucial role in mitotic spindle formation and elongation and is needed for the proper segregation of mitotic chromosomes during anaphase, but how it to induce chromosome segregation by regulating spindle movement is still not fully clear. The first reports indicated that depletion of EB1 in Drosophila leads to the formation of short spindles and short astral MTs [12]. In Xenopus egg extracts, EB1 was involved in spindle organization and chromosome segregation by interacting with XMAP215 [59]. In addition, together with APC, EB1 regulates chromosomal stability during mitosis [13].

Plants are prolific organisms covering the earth and are also irreplaceable partners for human beings. To enact morphogenesis, plants have evolved plant-specific MT arrays: cortical MTs, preprophase band, mitotic spindle, and phragmoplast. Plant microtubules (MTs) and MT-associated proteins (MAPs) are essential for fundamental morphogenesis, including controlling the direction of cell division and expansion, chromosome segregation, and cytokinesis. However, little is known about roles of EB1 in chromosome segregation in plants. Han et al. found that "partition-bundle" division would be happened in root-tip cells of wheat seedlings under the enhanced UV$\mathrm{B}$ radiation [60]. Also, the distribution of microtubules in wheat cells was disordered under the enhanced UV-B radiation [61]. But whether there is a correlation between "partition-bundle" division and disordered microtubules and whether EB1 plays a role in this process require to further be tested. But in Arabidopsis, EB1c is thought to function primarily during mitosis; it localizes to mitotic microtubule arrays in dividing cells and is sequestered in the nucleus during interphase. EB1c mutants exhibit lagging chromosomes during anaphase, suggesting that this protein subtype plays a role in early mitotic phases, regulating spindle positioning and chromosome segregation [11]. Moreover, in plant, the mutants of EB1 have twisting macroscopic phenotypes that caused by the change of MT array orientation from transverse to oblique in young elongating cells, indicating that these MAPs are involved in cortical MT organization to keep specific cell wide orientation, such as transverse to the long axis of the organ [62]. And the phenotype of the triple null mutant of the three EB1 genes includes defective spindle or phragmoplast organization [11]. In addition, EB1 mutants have mild defects in gravity and touch sensing in roots [62] [63].

\section{Acknowledgments}

This work was supported by the National Nature Science Foundation of China under Grant number 30671061 and the Natural Science of Shanxi Province under Grant number 20081101, 2014011028-5.

\section{References}

[1] Schuyler, S.C. and Pellman, D. (2001) Microtubule "Plus-End-Tracking Proteins": The End Is Just the Beginning. Cell, 105, 421-424. http://dx.doi.org/10.1016/S0092-8674(01)00364-6

[2] Akhmanova, A. and Steinmetz, M.O. (2008) Tracking the Ends: A Dynamic Protein Network Controls the Fate of Microtubule Tips. Nature Reviews Molecular Cell Biology, 9, 309-322. http://dx.doi.org/10.1038/nrm2369

[3] Akhmanova, A. and Hoogenraad, C.C. (2005) Microtubule Plus-End-Tracking Proteins: Mechanisms and Functions. Current Opinion in Cell Biology, 17, 47-54. http://dx.doi.org/10.1016/j.ceb.2004.11.001

[4] Honnappa, S., Gouveia, S.M., Weisbrich, A., Damberger, F.F., Bhavesh, N.S., Jawhari, H., Grigoriev, I., van Rijssel, F.J.A., Buey, R.M., Lawera, A., Jelesarov, I., Winkler, F.K., Wüthrich, K., Akhmanova, A. and Steinmetz, M.O. (2009) An EB1-Binding Motif Acts as a Microtubule Tip Localization Signal. Cell, 138, 366-376. http://dx.doi.org/10.1016/j.cell.2009.04.065

[5] Slep, K.C. (2010) Structural and Mechanistic Insights into Microtubule End-Binding Proteins. Current Opinion in Cell Biology, 22, 88-95. http://dx.doi.org/10.1016/j.ceb.2009.10.009

[6] Lansbergen, G. and Akhmanova, A. (2006) Microtubule Plus End: A Hub of Cellular Activities. Traffic, 7, $499-507$. http://dx.doi.org/10.1111/j.1600-0854.2006.00400.x

[7] Vaughan, K.T. (2005) TIP Maker and TIP Marker; EB1 as a Master Controller of Microtubule Plus Ends. The Journal 
of Cell Biology, 171, 197-200. http://dx.doi.org/10.1083/jcb.200509150

[8] Piehl, M. and Cassimeris, L. (2003) Organization and Dynamics of Growing Microtubule Plus Ends during Early Mitosis. Molecular Biology of the Cell, 14, 916-925. http://dx.doi.org/10.1091/mbc.E02-09-0607

[9] Cheerambathur, D.K. and Desai, A. (2014) Linked in: Formation and Regulation of Microtubule Attachments during Chromosome Segregation. Current Opinion in Cell Biology, 26, 113-122. http://dx.doi.org/10.1016/j.ceb.2013.12.005

[10] Zhai, Y., Kronebusch, P.J., Simon, P.M. and Borisy, G.G. (1996) Microtubule Dynamics at the G2/M Transition: Abrupt Breakdown of Cytoplasmic Microtubules at Nuclear Envelope Breakdown and Implications for Spindle Morphogenesis. The Journal of Cell Biology, 135, 201-214. http://dx.doi.org/10.1083/jcb.135.1.201

[11] Komaki1, S., Abe, T., Coutuer, S., Inzé, D., Russinova, E. and Hashimoto, T. (2010) Nuclear-Localized Subtype of End-Binding 1 Protein Regulates Spindle Organization in Arabidopsis. Journal of Cell Science, 123, 451-459. http://dx.doi.org/10.1242/jcs.062703

[12] Rogers, S.L., Rogers, G.C., Sharp, D.J. and Vale, R.D. (2002) Drosophila EB1 Is Important for Proper Assembly, Dynamics, and Positioning of the Mitotic Spindle. The Journal of Cell Biology, 158, 873-884. http://dx.doi.org/10.1083/jcb.200202032

[13] Green, R.A., Wollman, R. and Kaplan, K.B. (2005) APC and EB1 Function Together in Mitosis to Regulate Spindle Dynamics and Chromosome Alignment. Molecular Biology of the Cell, 16, 4609-4622. http://dx.doi.org/10.1091/mbc.E05-03-0259

[14] Cassirneris, L. (1993) Regulation of Microtubule Dynamic Instability. Cell Motility and the Cytoskeleton, 26, $275-281$. http://dx.doi.org/10.1002/cm.970260402

[15] Howard, J. and Hyman, A.A. (2009) Growth, Fluctuation and Switching at Microtubule plus Ends. Nature Reviews Molecular Cell Biology, 10, 569-574. http://dx.doi.org/10.1038/nrm2713

[16] Jiang, K. and Akhmanova, A. (2011) Microtubule Tip-Interacting Proteins: A View from Both Ends. Current Opinion in Cell Biology, 23, 94-101. http://dx.doi.org/10.1016/j.ceb.2010.08.008

[17] Mitchison, T. and Kirschner, M. (1984) Dynamic Instability of Microtubule Growth. Nature, 312, 237-242. http://dx.doi.org/10.1038/312237a0

[18] Masoud, K., Herzog, E., Chaboute, M.E. and Schmit, A.C. (2013) Microtubule Nucleation and Establishment of the Mitotic Spindle in Vascular Plant Cells. The Plant Journal, 75, 245-257. http://dx.doi.org/10.1111/tpj.12179

[19] Howard, J. and Hyman, A.A. (2003) Dynamics and Mechanics of the Microtubule plus End. Nature, 422, 753-758. http://dx.doi.org/10.1038/nature01600

[20] Maurer, S.P., Cade, N.I., Bohner, G., Gustafsson, N., Boutant, E. and Surrey, T. (2014) EB1 Accelerates Two Conformational Transitions Important for Microtubule Maturation and Dynamics. Current Biology, 24, 372-384. http://dx.doi.org/10.1016/j.cub.2013.12.042

[21] Su, L.K., Burrell, M., Hill, D.E., Gyuris, J., Brent, R., Wiltshire, R., Trent, J., Vogelstein, B. and Kinzler, K.W. (1995) APC Binds to the Novel Protein EB1. Cancer Research, 55, 2972-2977.

[22] Hayashi, I. and Ikura, M. (2003) Crystal Structure of the Amino-Terminal Microtubule-Binding Domain of EndBinding Protein 1 (EB1). The Journal of Biological Chemistry, 278, 36430-36434. http://dx.doi.org/10.1074/jbc.M305773200

[23] Komarova, Y., De Groot, C.O., Grigoriev, I., Gouveia, S.M., Munteanu, E.L., Schober, J.M., Honnappa, S., Buey, R.M., Hoogenraad, C.C., Dogterom, M., Borisy, G.G., Steinmetz, M.O. and Akhmanova, A. (2009) Mammalian End Binding Proteins Control Persistent Microtubule Growth. The Journal of Cell Biology, 184, 691-706. http://dx.doi.org/10.1083/jcb.200807179

[24] Steinmetz, M.O. and Akhmanova, A. (2008) Capturing Protein Tails by CAP-Gly Domains. Trends in Biochemical Sciences, 33, 535-545. http://dx.doi.org/10.1016/j.tibs.2008.08.006

[25] Galjart, N. (2010) Plus-End-Tracking Proteins and Their Interactions at Microtubule Ends. Current Biology, 20, 528537. http://dx.doi.org/10.1016/j.cub.2010.05.022

[26] Kumar, P. and Wittmann, T. (2012) +TIPs: SxIPping along Microtubule Ends. Trends in Cell Biology, 22, 418-428. http://dx.doi.org/10.1016/j.tcb.2012.05.005

[27] Maurer, S.P., Fourniol, F.J., Bohner, G., Moores, C.A. and Surrey, T. (2012) EBs Recognize a Nucleotide-Dependent Structural Cap at Growing Microtubule Ends. Cell, 149, 371-382. http://dx.doi.org/10.1016/j.cell.2012.02.049

[28] Su, L.K. and Qi, Y. (2001) Characterization of Human MAPRE Genes and Their Proteins. Genomics, 71, $142-149$. http://dx.doi.org/10.1006/geno.2000.6428

[29] Straube, A. and Merdes, A. (2007) EB3 Regulates Microtubule Dynamics at the Cell Cortex and Is Required for Myoblast Elongation and Fusion. Current Biology, 17, 1318-1325. http://dx.doi.org/10.1016/j.cub.2007.06.058 
[30] Bisgrove, S.R., Hable, W.E. and Kropf, D.L. (2004) + TIPs and Microtubule Regulation. The Beginning of the Plus End in Plants. Plant Physiology, 136, 3855-3863. http://dx.doi.org/10.1104/pp.104.051037

[31] Guo, L., Ho, C.M., Kong, Z., Lee, Y.R., Qian, Q. and Liu, B. (2009) Evaluating the Microtubule Cytoskeleton and Its Interacting Proteins in Monocots by Mining the Rice Genome. Annals of Botany, 103, 387-402. http://dx.doi.org/10.1093/aob/mcn248

[32] Chan, J., Calder, G.M., Doonan, J.H. and Lloyd, C.W. (2003) EB1 Reveals Mobile Microtubule Nucleation Sites in Arabidopsis. Nature Cell Biology, 5, 967-971. http://dx.doi.org/10.1038/ncb1057

[33] Chan, J., Calder, G., Fox, S. and Lloyd, C. (2005) Localization of the Microtubule End Binding Protein EB1 Reveals Alternative Pathways of Spindle Development in Arabidopsis Suspension Cells. Plant Cell, 17, 1737-1748. http://dx.doi.org/10.1105/tpc.105.032615

[34] Van Damme, D., Bouget, F.Y., Van Poucke, K., Inze, D. and Geelen, D. (2004) Molecular Dissection of Plant Cytokinesis and Phragmoplast Structure: A Survey of GFP-Tagged Proteins. The Plant Journal, 40, 386-398. http://dx.doi.org/10.1111/j.1365-313X.2004.02222.x

[35] Berrueta, L., Kraeft, S.K., Tirnauer, J.S., Schuyler, S.C., Chen, L.B., Hill, D.E., Pellman, D. and Bierer, B.E. (1998) The Adenomatous Polyposis Coli-Binding Protein EB1 Is Associated with Cytoplasmic and Spindle Microtubules. Cell Biology, 95, 10596-10601. http://dx.doi.org/10.1073/pnas.95.18.10596

[36] Tirnauer, J.S., Canman, J.C., Salmon, E.D. and Mitchison, T.J. (2002) EB1 Targets to Kinetochores with Attached, Polymerizing Microtubules. Molecular Biology of the Cell, 13, 4308-4316. http://dx.doi.org/10.1091/mbc.E02-04-0236

[37] Akhmanova, A. and Steinmetz, M.O. (2010) Microtubule +TIPs at a Glance. Journal of Cell Science, 123, 3415-3419. http://dx.doi.org/10.1242/jcs.062414

[38] Gard, D.L. and Kirschner, M.W. (1987) A Microtubule-Associated Protein from Xenopus Eggs That Specifically Promotes Assembly at the Plus-End. The Journal of Cell Biology, 105, 2203-2215. http://dx.doi.org/10.1083/jcb.105.5.2203

[39] Bieling, P., Kandels-Lewis, S., Telley, I.A., van Dijk, J., Janke, C. and Surrey, T. (2008) CLIP-170 Tracks Growing Microtubule Ends by Dynamically Recognizing Composite EB1/Tubulin-Binding Sites. The Journal of Cell Biology, 183, 1223-1233. http://dx.doi.org/10.1083/jcb.200809190

[40] Bu, W. and Su, L.K. (2001) Regulation of Microtubule Assembly by Human EB1 Family Proteins. Nature, 20, 31853192. http://dx.doi.org/10.1038/sj.onc.1204429

[41] Tirnauer, J.S. and Bierer, B.E. (2000) EB1 Proteins Regulate Microtubule Dynamics, Cell Polarity, and Chromosome Stability. The Journal of Cell Biology, 149, 761-766. http://dx.doi.org/10.1083/jcb.149.4.761

[42] Beinhauer, J.D., Hagan, I.M., Hegemann, J.H. and Fleig, U. (1997) Mal3, the Fission Yeast Homologue of the Human APC-Interacting Protein EB-1 Is Required for Microtubule Integrity and the Maintenance of Cell Form. The Journal of Cell Biology, 139, 717-728.

[43] Berrueta, L., Tirnauer, J.S., Schuyler, S.C., Pellman, D. and Bierer, B.E. (1999) The APC-Associated Protein EB1 Associates with Components of the Dynactin Complex and Cytoplasmic Dynein Intermediate Chain. Current Biology, 9, 425-428. http://dx.doi.org/10.1016/S0960-9822(99)80190-0

[44] Maurera, S.P., Bieling, P., Cope, J., Hoenger, A. and Surrey, T. (2011) GTP $\gamma$ S Microtubules Mimic the Growing Microtubule End Structure Recognized by End-Binding Proteins (EBs). Proceedings of the National Academy of Sciences of the United States of America, 108, 3988-3993. http://dx.doi.org/10.1073/pnas.1014758108

[45] Bieling, P., Laan, L., Schek, H., Munteanu, E.L., Sandblad, L., Dogterom, M., Brunner, D. and Surrey, T. (2007) Reconstitution of a Microtubule Plus-End Tracking System in Vitro. Nature, 450, 1100-1105. http://dx.doi.org/10.1038/nature06386

[46] Ligon, L.A., Shelly, S.S., Tokito, M. and Holzbaur, E.L. (2003) The Microtubule Plus-End Proteins EB1 and Dynactin Have Differential Effects on Microtubule Polymerization. Molecular Biology of the Cell, 14, 1405-1417. http://dx.doi.org/10.1091/mbc.E02-03-0155

[47] Lopus, M., Manatschal, C., Buey, R.M., Bjelic, S., Miller, H.P., Steinmetz, M.O. and Wilson, L. (2012) Cooperative Stabilization of Microtubule Dynamics by EB1 and CLIP-170 Involves Displacement of Stably Bound P(i) at Microtubule Ends. Biochemistry, 51, 3021-3030. http://dx.doi.org/10.1021/bi300038t

[48] Mimori-Kiyosue, Y., Grigoriev, I., Lansbergen, G., Sasaki, H., Matsui, C., Severin, F., Galjart, N., Grosveld, F., Vorobjev, I., Tsukita, S. and Akhmanova, A. (2005) CLASP1 and CLASP2 Bind to EB1 and Regulate Microtubule PlusEnd Dynamics at the Cell Cortex. The Journal of Cell Biology, 168, 141-153. http://dx.doi.org/10.1083/jcb.200405094

[49] Montenegro Gouveia, S., Leslie, K., Kapitein, L.C., Buey, R.M., Grigoriev, I., Wagenbach, M., Smal, I., Meijering, E., Hoogenraad, C.C., Wordeman, L., Steinmetz, M.O. and Akhmanova, A. (2010) In Vitro Reconstitution of the Functional Interplay between MCAK and EB3 at Microtubule Plus Ends. Current Biology, 20, 1717-1722. http://dx.doi.org/10.1016/j.cub.2010.08.020 
[50] Zimniak, T., Stengl, K., Mechtler, K. and Westermann, S. (2009) Phospho-Regulation of the Budding Yeast EB1 Homologue Bim1p by Aurora/Ipl1p. The Journal of Cell Biology, 186, 379-391. http://dx.doi.org/10.1083/jcb.200901036

[51] Iimori, M., Ozaki, K., Chikashige, Y., Habu, T., Hiraoka, Y., Maki, T., Hayashi, I., Obuse, C. and Matsumoto, T. (2012) A Mutation of the Fission Yeast EB1 Overcomes Negative Regulation by Phosphorylation and Stabilizes Microtubules. Experimental Cell Research, 318, 262-275. http://dx.doi.org/10.1016/j.yexcr.2011.11.006

[52] Ban, R., Matsuzaki, H., Akashi, T., Sakashita, G., Taniguchi, H., Park, S.Y., Tanaka, H., Furukawa, K. and Urano, T. (2009) Mitotic Regulation of the Stability of Microtubule Plus-End Tracking Protein EB3 by Ubiquitin Ligase SIAH-1 and Aurora Mitotic Kinases. The Journal of Biological Chemistry, 284, 8367-8381. http://dx.doi.org/10.1074/jbc.M109.000273

[53] Ferreira, J.G., Pereira, A.J., Akhmanova, A. and Maiato, H. (2013) Aurora B Spatially Regulates EB3 Phosphorylation to Coordinate Daughter Cell Adhesion with Cytokinesis. The Journal of Cell Biology, 201, 709-724. http://dx.doi.org/10.1083/jcb.201301131

[54] Komarova, Y.A., Huang, F., Geyer, M., Daneshjou, N., Garcia, A., Idalino, L., Kreutz, B., Mehta, D. and Malik, A.B. (2012) VE-Cadherin Signaling Induces EB3 Phosphorylation to Suppress Microtubule Growth and Assemble Adherens Junctions. Molecular Cell, 48, 914-925. http://dx.doi.org/10.1016/j.molcel.2012.10.011

[55] Gadde, S. and Heald, R. (2004) Mechanisms and Molecules of the Mitotic Spindle. Current Biology, 14, 797-805. http://dx.doi.org/10.1016/j.cub.2004.09.021

[56] vander Vaart, B., Akhmanova, A. and Straube, A. (2009) Regulation of Microtubule Dynamic Instability. Biochemical Society Transactions, 37, 1007-1013. http://dx.doi.org/10.1042/BST0371007

[57] Zhai, Y., Kronebusch, P.J., Simon, P.M. and Borisy, G.G. (1996) Microtubule Dynamics at the G2/M Transition: Abrupt Breakdown of Cytoplasmic Microtubules at Nuclear Envelope Breakdown and Implications for Spindle Morphogenesis. The Journal of Cell Biology, 135, 201-214. http://dx.doi.org/10.1083/jcb.135.1.201

[58] Schuyler, S.C. and Pellman, D. (2001) Search, Capture and Signal: Games Microtubules and Centrosomes Play. Journal of Cell Science, 114, 247-255.

[59] Kronja, I., Kruljac-Letunic, A., Caudron-Herger, M., Bieling, P. and Karsenti, E. (2009) XMAP215-EB1 Interaction Is Required for Proper Spindle Assembly and Chromosome Segregation in Xenopus Egg Extract. Molecular Biology of the Cell, 20, 2684-2696. http://dx.doi.org/10.1091/mbc.E08-10-1051

[60] Han, R., Wang, X., Yue, M. and Qi, Z. (2002) Effects of the Enhanced UV-B Radiation on the Body Cell Mitosis of the Wheat. Acta Genetica Sinica, 29, 537-541.

[61] Aihua, G., Limei, G., Yongfeng, L. and Han, R. (2010) Influence on Microtubule in Wheat Mesophyll Cell Exposed to Enhanced Ultraviolet-B Radiation and He-Ne Laser Irradiation. Guihaia, 30, 250-255.

[62] Bisgrove, S.R., Lee, Y.R., Liu, B., Peters, N.T. and Kropf, D.L. (2008) The Microtubule Plus-End Binding Protein EB1 Functions in Root Responses to Touch and Gravity Signals in Arabidopsis. Plant Cell, 20, 396-410. http://dx.doi.org/10.1105/tpc.107.056846

[63] Squires, S. and Bisgrove, S. (2013) The Microtubule-Associated Protein END BINDING1b, Auxin, and Root Responses to Mechanical Cues. Journal of Plant Growth Regulation, 32, 681-691. http://dx.doi.org/10.1007/s00344-013-9333-9 